\title{
Circular polariton currents with integer and fractional orbital angular momenta
}

\author{
E. S. Sedov $\odot,{ }^{1,2,3, *}$ V. A. Lukoshkin $\odot,{ }^{4,5}$ V. K. Kalevich $\odot^{4,5}$ P. G. Savvidis, ${ }^{1,2,6}$ and A. V. Kavokin ${ }^{1,2,5,7}$ \\ ${ }^{1}$ School of Science, Westlake University, 18 Shilongshan Road, Hangzhou 310024, Zhejiang Province, China \\ ${ }^{2}$ Institute of Natural Sciences, Westlake Institute for Advanced Study, 18 Shilongshan Road, Hangzhou 310024, Zhejiang Province, China \\ ${ }^{3}$ Vladimir State University named after A. G. and N. G. Stoletovs, Gorky str. 87, Vladimir 600000, Russia \\ ${ }^{4}$ Ioffe Institute, Russian Academy of Sciences, 26 Politekhnicheskaya, St. Petersburg 194021, Russia \\ ${ }^{5}$ Spin Optics Laboratory, St. Petersburg State University, 1 Unlianovskaya, St. Petersburg 198504, Russia \\ ${ }^{6}$ FORTH, Institute of Electronic Structure and Laser, Heraklion 71110, Crete, Greece \\ ${ }^{7}$ Russian Quantum Center, 100 Novaya Street, Skolkovo 143025, Moscow Region, Russia
}

(Received 21 September 2020; revised 2 December 2020; accepted 4 December 2020; published 22 January 2021)

\begin{abstract}
We study internal dynamics of exciton-polariton condensates created by the nonresonant optical pump in a cylindrical pillar microcavity with an ensemble of embedded quantum wells. The polariton condensates are intrinsically nonequilibrium systems: Their dissipative nature together with a spatial inhomogeneity of a potential landscape and localized pumping leads to formation of steady polariton flows. The gain-loss engineering consisting of a deliberate breaking of the rotational symmetry of the system makes the polariton flows controllable. We demonstrate the switching between the polariton current states characterized by both integer and fractional orbital angular momenta (OAM) by tuning the position and ellipticity of the pump spot. At a weak shift and a small ellipticity, the phase rather than the density of the exciton-polariton condensate is significantly affected. Then the polariton condensate is characterized by an integer OAM per particle coinciding with the topological charge of the polariton vortex state. We demonstrate experimentally the polariton current states with the topological charges from -3 to +2 . The further shift of the pump spot perturbs the azimuthal distribution of the polariton density and causes a jump of the phase of the condensate at the density deep. The mean orbital angular momentum characterizing such polariton condensate acquires fractional values. To describe the experimentally observed polariton current states, we propose a model based on the Gross-Pitaevskii equation projected onto the azimuthal states of the ring trap which treats the formation of fractional OAM states as a result of the coherent superposition of integer OAM states sustained due to the gain-loss balance in the system with a broken rotational symmetry.
\end{abstract}

DOI: 10.1103/PhysRevResearch.3.013072

\section{INTRODUCTION}

Light dramatically changes its properties when coupling to a resonant medium. The established platform for studying the effects induced by the light-matter coupling is specially designed two-dimensional semiconductor microcavities with embedded quantum wells (QWs) [1]. Being confined in a microcavity light modes hybridize with QW excitons and form new self-sufficient states called exciton polariton. One can refer to the flow of exciton polaritons as "liquid light" since its behavior is similar to that of a superfluid liquid $[2,3]$. If confined in a trap, the polariton liquid supports formation of internal flows analogous to currents of charge carriers in normal conductors or currents of Cooper pairs in superconducting circuits. The polariton currents in microcavities are

\footnotetext{
*evgeny_sedov@mail.ru

Published by the American Physical Society under the terms of the Creative Commons Attribution 4.0 International license. Further distribution of this work must maintain attribution to the author(s) and the published article's title, journal citation, and DOI.
}

characterized by the vector field of the current density $\mathbf{J}=$ $(i / 2)\left[\Psi(\mathbf{r}) \nabla \Psi^{*}(\mathbf{r})-\Psi^{*}(\mathbf{r}) \nabla \Psi(\mathbf{r})\right]$, where $\Psi(\mathbf{r})$ is the wave function of the polariton condensate.

Persistent currents are the intriguing phenomenon in quantum coherent systems. The nonreciprocity of the systems underlies the emergence of current states [4]. It implies breaking the equivalence of directions of propagation within the system whereby one of the directions becomes preferred. An extensive study of persistent currents was done in annular Bose-Einstein condensates of ultracold atoms. Breaking the reciprocity was achieved by applying a rotating barrier [5-7] or an artificial gauge field [8] induced, e.g., by an external laser beam [9] or by rotating the trapped condensate with a constant velocity [10]. A wide spectrum of approaches for the induction of a nonreciprocity was developed for photon transmission systems. Among them are the optomechanical coupling in microring resonators [11], refractive index modulation in waveguide ring resonators [12], and Kerr nonlinearity in photonic crystal circuits [13].

Exciton polariton condensates are known to be intrinsically strongly nonequilibrium systems $[14,15]$. In the stationary regime, they are governed by the requirements of a detailed balance between the pumping from an external source and 
losses of polaritons. This nonequilibrium nature of macroscopic polariton states offers new tools for breaking the reciprocity, namely the reservoir engineering [16-19] and the dissipation engineering [20,21]. It implies structuring the environment in a such manner that it supports the desirable behavior of the system [16]. It can be considered as a local modification of an effective complex potential which affects upon the nonlocal gain and dissipation, so that the system becomes chiral. This results in the appearance of intrinsic current states of polaritons.

The conventional way to the characterization of enclosed internal currents of exciton polaritons is using their orbital degree of freedom which is the orbital angular momentum (OAM) per particle. The presence of internal currents in the exciton-polariton condensate is reflected in a nonzero OAM of light emitted by the condensate. The orbital angular momentum per particle $\ell$ may be found from the polariton current density $\mathbf{J}$ as follows:

$$
\ell=\frac{L_{z}}{N}=\frac{1}{N} \int_{-\infty}^{\infty}\left(x J_{y}-y J_{x}\right) d \mathbf{r}
$$

where $N=\int_{-\infty}^{\infty}|\Psi(\mathbf{r})|^{2} d \mathbf{r}$ is the number of polaritons in the condensate, and the integral in the right-hand side is the actual OAM $L_{z}$.

A nonzero OAM $\ell$ is often confused with the winding number $m$ which is the characteristic of vortex states. It is also known as the vortex topological charge. To distinguish between these two crucial characteristics, one can represent the complex wave function of the polariton condensate in the form $\Psi(\mathbf{r})=\rho^{1 / 2}(\mathbf{r}) \exp [i \varphi(\mathbf{r})]$, where $\rho(\mathbf{r})$ and $\varphi(\mathbf{r})$ are the spatial distributions of the density and the phase of the condensate. A vortex state is characterized by the phase singularity at the point of vanishing density referred to as the vortex core [22]. The winding number $m$ characterizes the circulation of the gradient of the condensate phase around the singularity point. It is introduced as follows:

$$
m=\frac{1}{2 \pi} \oint \nabla \varphi(\mathbf{r}) d \mathbf{l},
$$

where $d \mathbf{l}$ is the change along the closed path around the singularity. Physically, $m$ is a measure of the velocity of the phase variation around the singularity. The positive $m$ corresponds to the counterclockwise variation while the negative $m$ stands for the clockwise variation [23]. Since the scalar wave function of the condensate is required to be single valued, the phase may only change by a multiple of $2 \pi$ as one goes around the core, so that the winding number $m$ is an integer.

In an infinite isotropic superfluid, vortices possess the azimuthally homogeneous density distribution with the central node. In this case, values of the characteristic OAM per particle $\ell$ and the winding number $m$ coincide, indicating that the internal current in the superfluid is accompanied by the circulation of the phase gradient around the singularity. The creation of polariton vortices using the optical control was demonstrated, e.g., in [24-26]. In a general case, however, the values of $\ell$ and $m$ do not necessarily match. In particular, they frequently mismatch in chiral systems generated by means of the reservoir engineering. Moreover, due to the azimuthal density variation, the OAM $\ell$ is not forced to be quantized and it can acquire any real values $[27,28]$. The origin of the states with fractional OAM, known, e.g., in optics [29,30] and ultracold atoms [6,7], is a distortion in the annular intensity (or density) distribution accompanied by the phase discontinuity. In optics, the latter is induced by using specially designed vortex lenses [31] or spiral plates [32]. In Bose-Einstein condensates of ultracold atoms the density depletion in the azimuthal direction is induced by the local defect, that is, a so-called weak link [6,7].

The states with fractional OAM are also known in polaritonics. The spin (polarization) degree of freedom is essential for the formation of such a state. In Ref. [33] half-quantum polariton vortices with the average OAM per particle $|\ell|=0.5$ were proposed theoretically. These states represent a spinor polariton superliquid where a quantized vortex is present for one spin (polarization) component and there is no vortex in another spin (polarization) component. References [34-36] report on the experimental evidence of such states. Reference [37] reports on another type of a half-OAM polariton state involving the polarization degree of freedom. It exists in the ring geometry close to that considered by us here. The inversion of the circular polarization when moving around the ring is a characteristic feature of that state.

In the present work we limit ourselves to scalar polariton fields, i.e., we leave the spinor polariton condensates and spin-orbit interaction effects beyond the scope of our consideration. To generate integer and fractional OAM states of polaritons, we create an effective complex potential landscape which enables modulation of the density of the polariton condensate and introduce the chirality to the system. As we show both theoretically and experimentally, in the presence of the induced chirality and polariton-polariton interactions, the circular superfluid current states with nonzero OAM, $\ell \neq 0$, may be formed in driven-dissipative polariton condensates even in the absence of vorticity, $m=0$.

\section{PROJECTED GENERALIZED GROSS-PITAEVSKII EQUATION}

We model persistent polariton currents in a ring trap with use of the generalized Gross-Pitaevskii equation (gGPE) for the polariton condensate wave function $\Psi(t, \mathbf{r})[3]$ :

$$
\begin{aligned}
i \hbar \partial_{t} \Psi= & {\left[-\frac{\hbar^{2}}{2 M} \nabla^{2}+V(\mathbf{r})+\alpha|\Psi|^{2}+\alpha_{\mathrm{R}} n_{\mathrm{R}}\right] \Psi } \\
& +\frac{i \hbar}{2}\left(R n_{\mathrm{R}}-\gamma\right) \Psi,
\end{aligned}
$$

where $M$ is the effective mass of a polariton. $V(\mathbf{r})=V_{\mathrm{c}}(r)+$ $\delta V(\mathbf{r})$ is the stationary potential combining the axially symmetric trapping potential defined by the edge of the pillar $V_{\mathrm{c}}(r)$ and the symmetry-breaking term $\delta V(\mathbf{r})$ responsible for the reduction of the axial symmetry of the pillar. The parameters $\alpha$ and $\alpha_{\mathrm{R}}$ are accounting for the polariton-polariton and polariton-exciton interaction, respectively. The repulsive interaction of polaritons with the exciton reservoir is responsible for the formation of the maximum of the effective potential for the polaritons under the pump spot, where the reservoir is predominantly localized. The height of the potential is proportional to the concentration of excitons in the reservoir and it can be further increased by involving optically inactive 
(dark) exciton states [38]. The right parentheses in Eq. (3) are responsible for the balance of gain and losses in the polariton condensate. $R$ is the stimulated scattering rate from the reservoir to the polariton mode; $\gamma$ is the decay rate of polaritons.

The polariton state is fed from the reservoir of excitons, which is excited by the nonresonant optical pump. The reservoir density $n_{\mathrm{R}}(t, \mathbf{r})$ obeys the rate equation,

$$
\partial_{t} n_{\mathrm{R}}=P(\mathbf{r})-\left(\gamma_{\mathrm{X}}+R|\Psi|^{2}\right) n_{\mathrm{R}},
$$

where $\gamma_{\mathrm{x}}$ is the exciton decay rate. $P(\mathbf{r})$ is the pump intensity which we take in the form of a Gaussian, $P(\mathbf{r}) \propto$ $\exp \left[-\left(x-x_{\mathrm{p}}\right)^{2} / 2 w_{x}^{2}+\left(y-y_{\mathrm{p}}\right)^{2} / 2 w_{y}^{2}\right]$, shifted from the center of the pillar by a vector $\mathbf{r}_{\mathrm{p}}=\left(x_{\mathrm{p}}, y_{\mathrm{p}}\right) . w_{x, y}$ are the widths of the pump spot in $x$ and $y$ directions. We introduce the ellipticity of the pump beam as $s=w_{y} / w_{x}$. For the further consideration, it is convenient to represent the pump as $P(\mathbf{r})=P_{\mathrm{s}}(r)+\delta P(\mathbf{r})$, where $P_{\mathrm{s}}(r)$ is the azimuthally symmetric component given by $P_{\mathrm{s}}(r)=\left.P(\mathbf{r})\right|_{\mathbf{r}_{\mathrm{p}}=(0,0), w_{x}=w_{y}} \propto$ $\exp \left[-\left(x^{2}+y^{2}\right) / 2 w_{x}^{2}\right]$, and $\delta P(\mathbf{r})$ is the symmetry-breaking perturbation of the pump.

Below we assume that the dynamics of excitons in the reservoir is fast with respect to the dynamics of the condensate, which allows us to exclude Eq. (4) from the model following the approach developed in [39]. We take the reservoir density in the form,

$$
n_{\mathrm{R}}(t, \mathbf{r}) \simeq \frac{P(\mathbf{r})}{\gamma_{\mathrm{X}}+R|\Psi|^{2}} \approx \frac{P(\mathbf{r})}{\gamma_{\mathrm{X}}}-\frac{P(\mathbf{r}) R|\Psi|^{2}}{\gamma_{\mathrm{X}}^{2}},
$$

where the first term describes the creation of the exciton cloud by the optical pump, and the second term is responsible for the depletion of the reservoir.

\section{A. Azimuthally symmetric problem}

Let us first consider the azimuthally symmetric linear problem, assuming $\delta V(\mathbf{r})=\delta P(\mathbf{r})=0$ and setting $|\Psi|^{2} \rightarrow$ 0 . In a narrow ring, the confining energy in the radial direction significantly exceeds the kinetic energy in the azimuthal direction. This allows one to solve the radial problem first, next treating the azimuthal problem as a perturbation [14,40,41]. In this case, Eq. (3) enables separation and factorization of the polariton wave function in cylindrical coordinates $\mathbf{r}=(r, \theta)$ as $\Psi_{m, n}(t, \mathbf{r})=\Psi_{m, n}(\mathbf{r}) \exp \left(-i E_{m, n} t\right)=$ $\Upsilon_{n}(r) \exp \left[i\left(m \theta-E_{m, n} t\right)\right]$. Here $E_{m, n}=E_{r}^{(n)}+E_{\theta}^{(m, n)}$ is the complex eigenenergy of the $(n, m)$ state, where $n$ and $m$ are the radial and azimuthal quantum numbers, respectively. $E_{r}^{(n)}$ and $E_{\theta}^{(m, n)}$ are the eigenvalues of the radial and azimuthal problems. The radial component of the wave function obeys the following stationary equation:

$$
\begin{aligned}
E_{r}^{(n)} \Upsilon_{n}= & {\left[-\frac{\hbar^{2}}{2 M} \nabla_{r}^{2}+V_{\mathrm{c}}(r)+\frac{\alpha_{\mathrm{R}} P_{\mathrm{s}}(r)}{\gamma_{\mathrm{x}}}\right] \Upsilon_{n} } \\
& +\frac{i \hbar}{2}\left(\frac{R P_{\mathrm{s}}(r)}{\gamma_{\mathrm{X}}}-\gamma\right) \Upsilon_{n},
\end{aligned}
$$

where $\nabla_{r}^{2}=\partial_{r r}^{2}+r^{-1} \partial_{r}$. In a narrow ring, the vast majority of polaritons occupies the lowest radial mode, $n=1$. This allows one to exclude the highest modes from further consideration keeping only $\Upsilon_{1}(r)$. In further sections we will demonstrate an experimental evidence of the validity of this

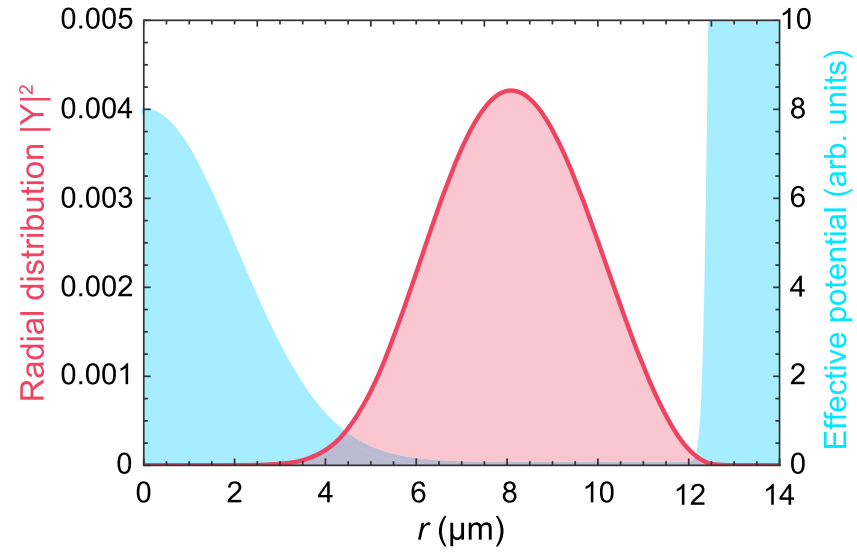

FIG. 1. The radial distribution of the density of the polariton condensate. The blue shaded region denotes the effective potential formed by the cloud of reservoir excitons (left hill) and the edge of the pillar (right wall).

assumption. Below we omit the index " $n$ " for the ease of notation, setting $\Psi_{m}(\mathbf{r}) \equiv \Psi_{1, m}(\mathbf{r}), \Upsilon(r) \equiv \Upsilon_{1}(r), E_{m} \equiv E_{1, m}$, and $E_{\theta}^{(m)} \equiv E_{\theta}^{(1, m)}$. The contribution from the azimuthal component of the wave function to the eigenenergy of the ground radial mode is found as $E_{\theta}^{(m)}=\left(\hbar^{2} m^{2} / 2 M\right) \int_{0}^{\infty}|\Upsilon|^{2} r^{-1} d r$. The radial distribution of the polariton condensate calculated for the set of parameters given in [42] is shown in Fig. 1.

\section{B. Projection of gGPE onto the azimuthally symmetric basis}

To visualize the effect of the symmetry breaking, we now project the gGPE (3) onto the basis of $\Psi_{m}(\mathbf{r})$. First, we substitute the factorized wave function $\Psi(t, \mathbf{r})=\Upsilon(r) \Phi(t, \theta)$ into Eq. (3) and, after averaging over $r$, we obtain the equation for the azimuthal component $\Phi(t, \theta)$ :

$$
\begin{aligned}
\partial_{t} \Phi(t, \theta)= & -i \hbar^{-1} \hat{T} \Phi(t, \theta)-\left[i U_{1}(\theta)-I_{1}(\theta)\right] \Phi(t, \theta) \\
& -\left[i U_{2}(\theta)-I_{2}(\theta)\right]|\Phi(t, \theta)|^{2} \Phi(t, \theta),
\end{aligned}
$$

where the kinetic energy operator $\hat{T}$ and the coefficients depending on the angle $\theta$ are given by

$$
\begin{aligned}
\hat{T} & =E_{r}-\frac{\hbar^{2}}{2 M}\left(\int_{0}^{\infty}|\Upsilon(r)|^{2} r^{-1} d r\right) \frac{\partial^{2}}{\partial \theta^{2}}, \\
U_{1}(\theta) & =\frac{1}{\hbar} \int_{0}^{\infty}\left[\delta V(\mathbf{r})+\frac{\alpha_{\mathrm{R}}}{\gamma_{\mathrm{X}}} \delta P(\mathbf{r})\right]|\Upsilon(r)|^{2} r d r, \\
I_{1}(\theta) & =\frac{R}{2 \gamma_{\mathrm{X}}} \int_{0}^{\infty} \delta P(\mathbf{r})|\Upsilon(r)|^{2} r d r, \\
U_{2}(\theta) & =\frac{1}{\hbar} \int_{0}^{\infty}\left(\alpha-\frac{R \alpha_{\mathrm{R}} P(\mathbf{r})}{\gamma_{\mathrm{X}}^{2}}\right)|\Upsilon(r)|^{4} r d r, \\
I_{2}(\theta) & =-\frac{R^{2}}{2 \gamma_{\mathrm{X}}^{2}} \int_{0}^{\infty} P(\mathbf{r})|\Upsilon(r)|^{4} r d r .
\end{aligned}
$$

The coefficients $U_{1}(\theta)$ and $I_{1}(\theta)$ describe symmetry-breaking contributions coming from the shift of the pump spot and from the built-in anisotropy of the stationary potential. $U_{2}(\theta)$ and $I_{2}(\theta)$ are responsible for the effect of interactions.

We now expand the azimuthal wave function component $\Phi(t, \theta)$ in terms of the spectrum of eigenfunctions of the 
azimuthally symmetric problem as follows:

$$
\Phi(t, \theta)=\sum_{m \in \mathbb{Z}} \psi_{m}(t) e^{i m \theta},
$$

where $\psi_{m}(t)$ are the time-dependent complex coefficients of the expansion. To further simplify the problem, we expand the coefficients (8) in the following series:

$$
U_{j}=\sum_{m \in \mathbb{Z}} U_{j}^{(m)} e^{i m \theta}, \quad I_{j}=\sum_{m \in \mathbb{Z}} I_{j}^{(m)} e^{i m \theta},
$$

where $j=1,2$. Substituting the expansions (9) and (10) into (7), we finally obtain the following system of the first-order differential equations for the coefficients $\psi_{m}(t)$ :

$$
\begin{aligned}
\partial_{t} \psi_{m}= & -i \hbar^{-1} E_{m} \psi_{m}-\sum_{m^{\prime} \in \mathbb{Z}}\left(i U_{1}^{\left(m^{\prime}\right)}-I_{1}^{\left(m^{\prime}\right)}\right) \psi_{m-m^{\prime}} \\
& -\sum_{m^{\prime} \in \mathbb{Z}} \sum_{m^{\prime \prime} \in \mathbb{Z}} \sum_{m^{\prime \prime \prime} \in \mathbb{Z}} \sum_{m^{\prime \prime \prime \prime} \in \mathbb{Z}} \delta_{m+m^{\prime \prime \prime \prime}, m^{\prime}+m^{\prime \prime}+m^{\prime \prime \prime}}\left(i U_{2}^{\left(m^{\prime}\right)}-I_{2}^{\left(m^{\prime}\right)}\right) \psi_{m^{\prime \prime \prime}}^{*} \psi_{m^{\prime \prime \prime}} \psi_{m^{\prime \prime}}
\end{aligned}
$$

We can now define the population of the polariton state $N(t)$ and the orbital angular momentum per particle $\ell(t)$ via the expansion (9) as follows:

$$
\begin{aligned}
N(t) & =\sum_{m \in \mathbb{Z}}\left|\psi_{m}(t)\right|^{2}, \\
\ell(t) & =\frac{1}{N(t)} \sum_{m \in \mathbb{Z}} m\left|\psi_{m}(t)\right|^{2} .
\end{aligned}
$$

It is convenient to characterize OAM by calculating the imbalance of contributions from the clockwise and the anticlockwise polariton currents:

$$
\ell(t)=\frac{1}{N(t)} \sum_{m \in \mathbb{N}} m\left(\left|\psi_{m}(t)\right|^{2}-\left|\psi_{-m}(t)\right|^{2}\right) .
$$

The second term in the right-hand side of Eq. (11) can be considered as a source of internal circular currents in a polariton condensate. Due to the chirality induced by the azimuthal asymmetry of the pump and the effective potential the orthogonal spectral components, $m$ and $-m$, are pumped with different rates. They all are subjects to different potential landscapes. This induces an imbalance in populations of the winding states of opposite direction and leads to the appearance of polariton flows of preferred directions. The broken spatial symmetry of the system is responsible for the generation of polariton currents. It is important to note that its effect is different for different spectral components $m$. That is why the broken symmetry cannot be treated as a homogeneous gauge field, as it was done, e.g., in atomic condensates for particle flows induced by rotation of a weak link or a trap at a constant velocity $[6,7,27]$. The localized pump is at the origin of the nonlocal interplay between pump and decay. The local measure of the pump-decay balance is the divergence of the flux $D=\nabla \mathbf{J}$ [44], which for the azimuthal component reduces to

$$
D_{\theta}(\theta)=\sum_{m \in \mathbb{N}} \sum_{m^{\prime} \in \mathbb{N}} m\left(m^{\prime}-m\right) \psi_{m^{\prime}}^{*} \psi_{m} e^{i\left(m-m^{\prime}\right) \theta} .
$$

$D_{\theta}>0$ for the gain region and $D_{\theta}<0$ for the loss region. In the steady-state regime where the overall loss is exactly compensated by the pumping, the following condition is fulfilled: $\int_{0}^{2 \pi} D_{\theta} d \theta=0$.

The proposed model is valid at a moderate pump power where the system is in the nonlinear regime. As Ref. [45] shows, in this regime the lobe patterns transform to the (a)

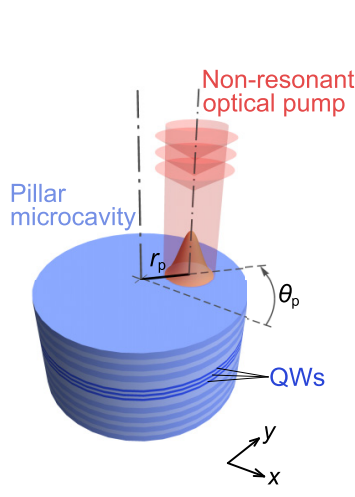

(b)

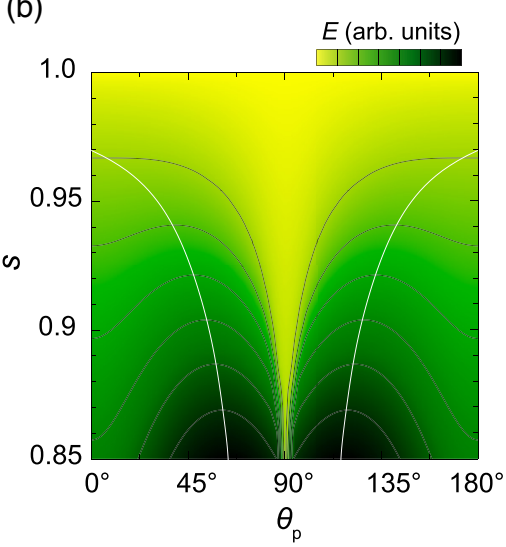

(c)

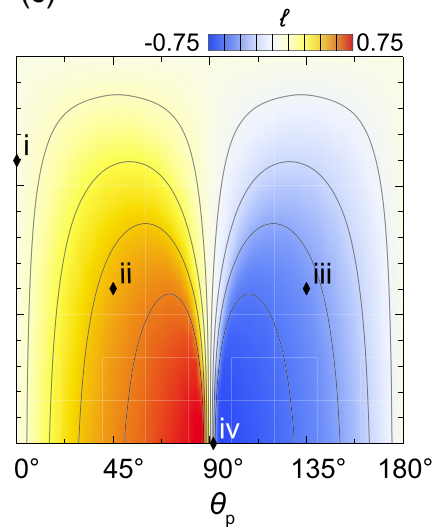

(d)

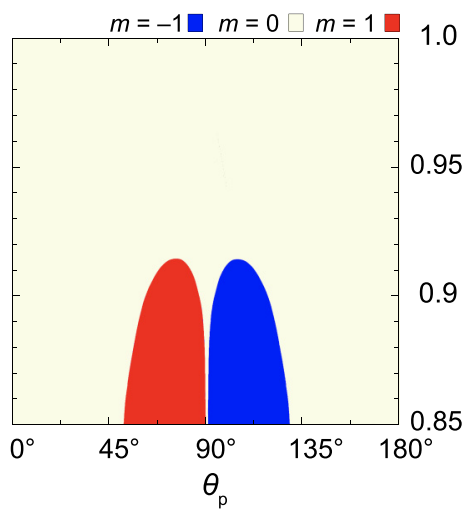

FIG. 2. Schematic of excitation of polaritons with a nonresonant optical pump in a pillar microcavity with embedded quantum wells (a). Energy $E(\mathrm{~b}), \mathrm{OAM} \ell(\mathrm{c})$, and topological charge $m(\mathrm{~d})$ of the polariton condensate as functions of the azimuthal coordinate $\theta_{\mathrm{p}}$ and the ellipticity $s$ of the pump spot. The pump spot is shifted by $r_{\mathrm{p}}=0.5 \mu \mathrm{m}$ along one of the radial directions. The equivalue contours in (b) and (c) are the guides for the eye. The white curves in (b) indicate the positions of the energy maxima as functions of $s, E_{\max }(s)$. Black diamonds numbered from $i$ to iv in panel (c) indicate the parameters used in Fig. 3. 
i

(a)

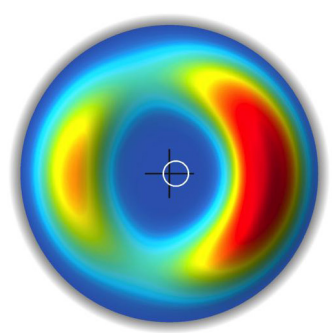

(b)

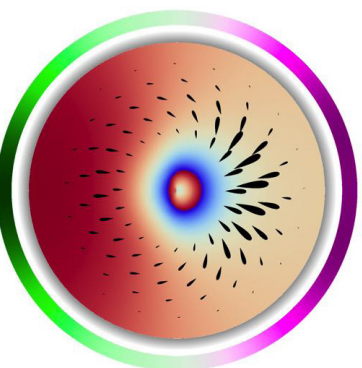

(c)

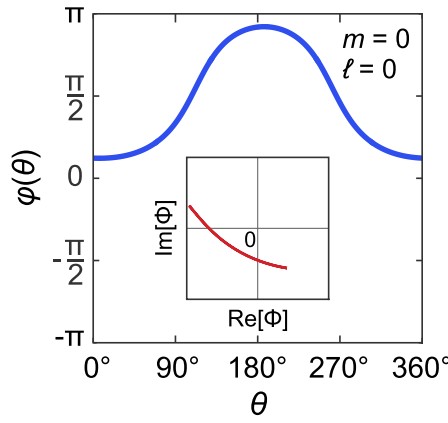

ii

(d)

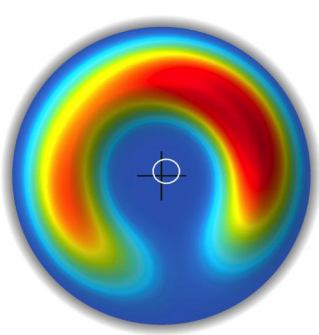

(e)

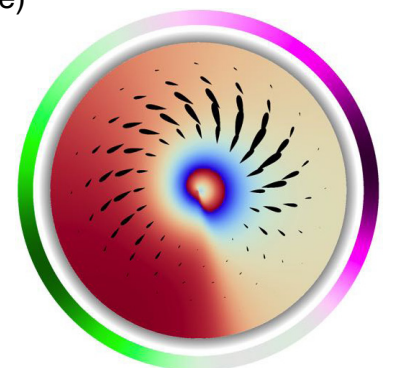

(f)

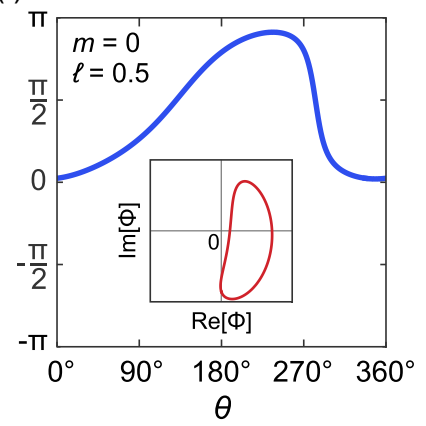

iii

(g)

(h)
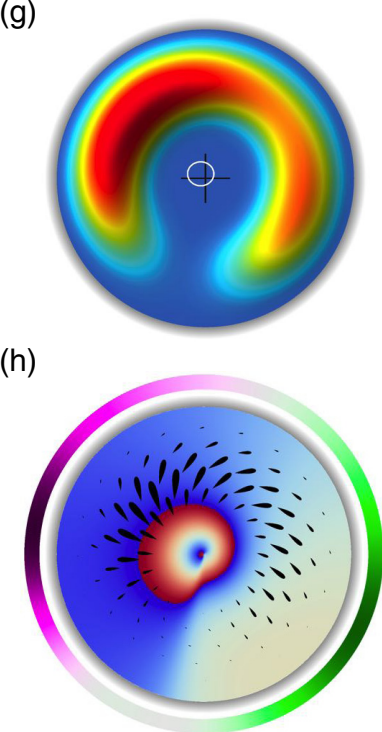

(j)

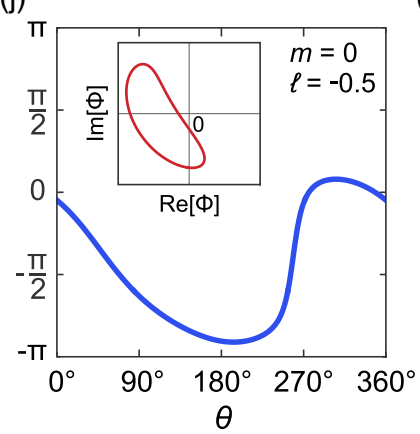

iv

(k)

(I)
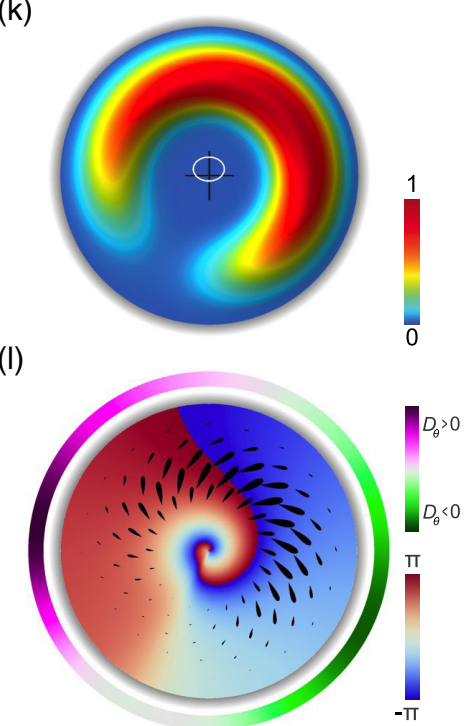

(m)

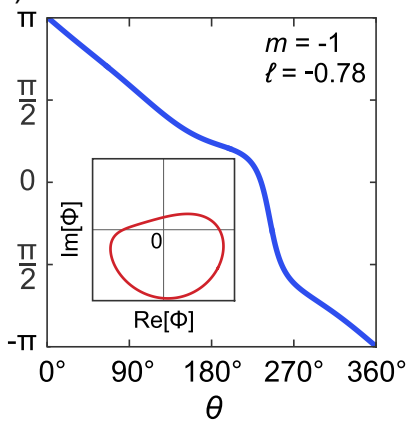

FIG. 3. The simulated spatial distribution of the density (upper panels) and the phase (middle panels) as well as the azimuthal distribution of the phase (lower panels) of the polariton condensates at different positions and ellipticities of the pump corresponding to those indicated by the black diamonds in Fig. 2 numbered from i to iv: $\theta_{\mathrm{p}}=0$ and $s=0.96$ (a) $-(\mathrm{c}), \theta_{\mathrm{p}}=45^{\circ}$ and $s=0.91(\mathrm{~d})-(\mathrm{f}), \theta_{\mathrm{p}}=135^{\circ}$ and $s=0.91(\mathrm{~g})-(\mathrm{j})$, $\theta_{\mathrm{p}}=91^{\circ}$ and $s=0.85(\mathrm{k})-(\mathrm{m})$. The black cross and the white ellipse in the upper panels indicate the center of the pillar and the position and the ellipticity of the pump spot, respectively. The black drops in the middle panels show the vector field of the current density $\mathbf{J}$. The purple-green circles around the meaning region indicate the local measure of the gain-loss balance: $D_{\theta}>0$ (purple) and $D_{\theta}<0$ (green). The inserts in the lower panels show the parametric relationship of the real and imaginary parts of the azimuthal component of the polariton wave function $\Phi(\theta)$ that serves to distinguish vortex $(m \neq 0)$ and nonvortex $(m=0)$ states: For the former, the point $(0,0)$ is inside the parametric contour.

crescent patterns and the separation of the radial and azimuthal components of the polariton wave function becomes possible.

Another limitation of the applicability of the proposed model is the polariton lifetime which should be large enough for the formation of circular polariton condensates around the pump-induced potential maximum. In contrast to the shortliving polaritons considered, e.g., in [46], where the condensate is formed under the ring shape pump spot, the long-living polaritons form condensates beyond the pump spot in the minimum of the potential landscape. The recent achievements in the growth of high-Q microcavities allow for experimental studies of polaritons having lifetimes of the order of hundreds of picosecond $[47,48]$. Nevertheless, even a more modest lifetime of up to a few tens of picosecond [49] is sufficient for the condensate to be formed outside the pump spot.

Our model can be adapted for another geometry of a possible experiment where the pump-induced potential and the stationary potential swap. To create an effective potential trap of a required landscape, instead of shaping polariton structures by means of deep etching of microcavities [50-52] one can use an all-optical approach. Optical traps created by nonresonant pumping have proven themselves to be an efficient controllable tool for generation of spatially localized polariton condensates [53-55]. In combination with the central potential maximum, regardless of its origin, the ring-shape optical traps allow creating a chiral surrounding required for the induction of circular polariton currents.

\section{POLARITON CURRENT STATES WITH FRACTIONAL OAM}

We consider the steady-state regime reached by an excitonpolariton condensate after its initial evolution and sustained by the balance of the spatially localized gain and losses. Since the pump spot plays the role of both the symmetry-breaking potential barrier and the spatially localized pump, the height of the barrier is correlated with the gain of the condensate 
and it cannot be considered as an independent parameter. The azimuthally symmetric states are achieved if the pump spot is situated right at the center of the pillar, $\mathbf{r}_{\mathrm{p}}=0$, and in the case of no ellipticity of the pump beam, $s=1$. Such states are characterized by well-defined integer OAM. Herewith, the states with $\ell \neq 0$ are vortex states with the topological charge $m=\ell$. The vortex states either appear spontaneously [55] or are imprinted by an external impact force [14], e.g., by a resonant short laser pulse [2]. The presence of the nonlinearity caused by interactions affects the energy of the final state rather than its OAM.

Breaking the rotational invariance itself causes the mixing of the polariton vortex states with different winding numbers $m$. In the presence of the nonlinearity, this leads to the formation of the current states with noninteger OAM. To break the rotational symmetry, we take the pump beam with an elliptical cross section $(s \neq 1)$ shifted from the center of the pillar by $\mathbf{r}_{\mathrm{p}} \neq 0$. No variation of the stationary potential is considered, $\delta V(\mathbf{r})=0$. In the further consideration, we use the ellipticity $s$, the azimuthal $\theta_{\mathrm{p}}$, and the radial $r_{\mathrm{p}}$, shifts of the pump as the control parameters allowing one to manipulate the polariton current states. A schematic of excitation of an exciton-polariton condensate in a micropillar is shown in Fig. 2(a). The color maps Figs. 2(b)-2(d) show the energy (b), OAM (c), and topological charge (d) of the polariton current state as functions of the azimuthal shift and the ellipticity of the pump spot at the radial shift taken as $r_{\mathrm{p}}=0.5 \mu \mathrm{m}$. The same patterns are repeated for the angles from $180^{\circ}$ to $360^{\circ}$. The other parameters are given in [42].

The appearance of fractional OAM ground polariton states is evident from the figure. Herewith, the value of $\ell$ is a subject to controllability. The polariton condensate OAM can be both positive (the pump spot is in quadrants I and III) and negative (the pump spot is in quadrants II and IV). The larger the achievable absolute value of $\ell$ is, the smaller the parameter of the ellipticity of the pump spot $s$. We would like to emphasize the different behavior of both dependencies of $E$ and $\ell$ on the azimuthal shift $\theta_{\mathrm{p}}$ around $\theta_{\mathrm{p}}=0^{\circ}$ and $90^{\circ}$. Although at both angles the azimuthal symmetry is broken due to the shift of the pump, the system is not chiral as it possesses an axial symmetry with respect to the axes of the pump ellipse. The parameter $s<1$ indicates that the major axis of the ellipse is parallel to the $x$ axis. When the azimuthal shift of the pump is $\theta_{\mathrm{p}}=0^{\circ}$, the axis of symmetry splits the pump ellipse along its major axis, while for the shift of $\theta_{\mathrm{p}}=90^{\circ}$, the minor axis is the axis of symmetry. Since the effective potential from the pump is more slopping along the major axis, the overlap of pump with the condensate is the highest in this direction. A larger angular size of the elliptic pump spot along the major axis causes a stronger sensitivity of the polariton state to a small shift along this axis.

In Fig. 3 we show examples of the characteristic polariton current states with fractional OAM. In column i [Figs. 3(a)3(c)] the polariton state with $\ell=0$ is shown, that is, obtained with the major axis of the pump spot being parallel to the direction of the shift of the pump. Since the system is nonchiral, the clockwise and counterclockwise current states are pumped equally. In the conservative linear limit, the resulting steady state could be considered as a standing wave. Here we deal with the nonlinear eigenstate, which due to the spatially localized gain and homogeneous loss possesses internal currents; see Fig. 3(b). The currents are centrifugal due to the maximum of the effective potential originated by the pump near the center of the pillar. Due to the shift of the pump, the currents flow along the edge of the pillar which results in the variation of the phase of the polariton condensate in space. The local gain region in the azimuthal direction $\left(D_{\theta}>0\right)$ on the side of the shifted pump is replaced by the loss region $\left(D_{\theta}<0\right)$ on the opposite side of the pillar; see the purplegreen ring around Fig. 3(b). The phase varies smoothly in the azimuthal direction and does not exhibit any sharp jumps; see Fig. 3(c).

The polariton current states with OAM $\ell=0.5$ and $\ell=-0.5$ are shown in the columns ii and iii in Fig. 3,

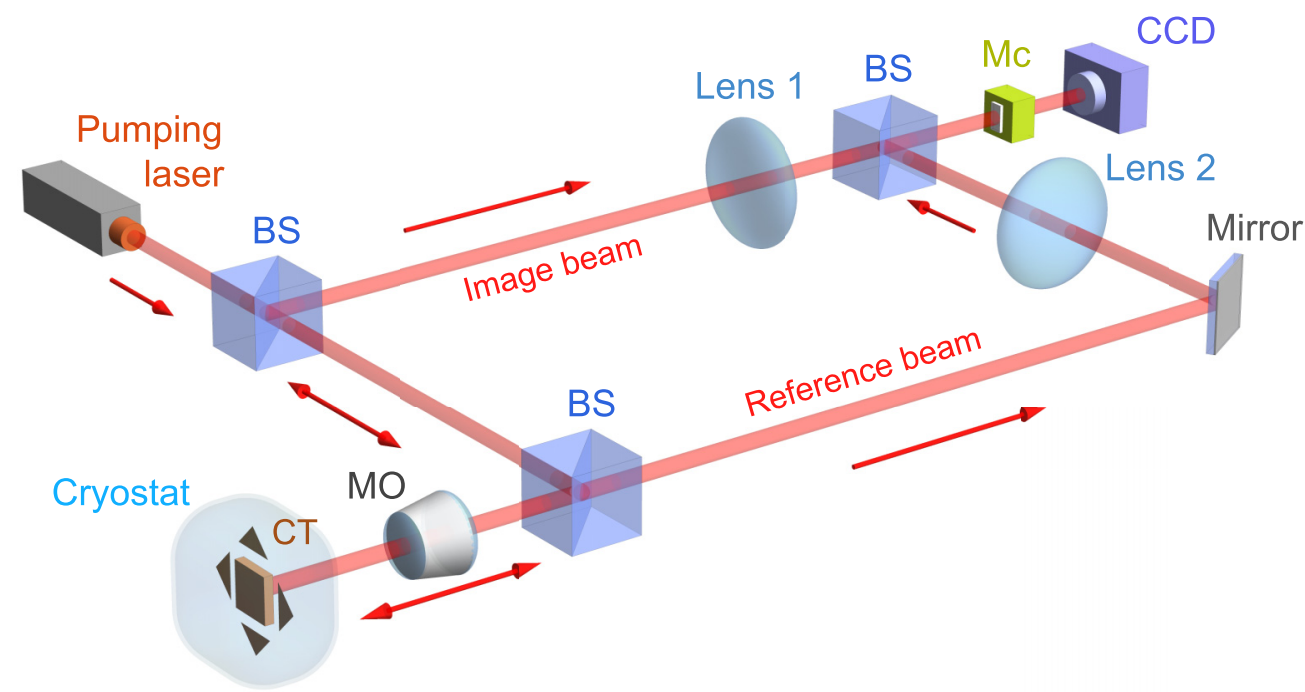

FIG. 4. Schematic of the experimental setup for photoluminescence and interferometry measurements. The following abbreviations are used in the figure: beam splitter (BS), monochromator (Mc), microscope objective (MO), coordinate table with the sample (CT). The red arrows indicate directions of propagation of the light beam along the optical track. 
respectively. The peculiarity of the fractional OAM states is the slit in the azimuthal density distribution. The azimuthal shift of the pump spot is about $45^{\circ}$ and $135^{\circ}$ in Figs. 3(d) and $3(\mathrm{~g})$, respectively. Since the equivalence of the clockwise and counterclockwise directions is broken in these cases, the states are characterized by internal currents of preferred azimuthal directions. The centrifugal runaway of polaritons is present also. The phase changes in the azimuthal direction, herewith the monotonic variation beyond the polariton density slit coexists with the sharp jump of the phase in the density minimum. The jump compensates the phase change, so that the phase gained by a polariton during one full pass around the pillar is zero. We would like to underline that although in both cases circular polariton currents are observed, these are not vortex states. The winding number for these states is calculated to be $m=0$. The inserts in Figs. 3(f) and 3(j) allow one to distinguish these current states from the vortex states, as the coordinate origin on the phase plane $(\operatorname{Re}[\Phi], \operatorname{Im}[\Phi])$ is beyond the area circumscribed by the closed curves characterizing the azimuthal component of the polariton wave function.

In the phase diagram in Fig. 2(d) we predict the existence of both vortex and no-vortex states with nonzero OAM. The polariton current state acquires vorticity with the increasing OAM. The value of $\ell$ when the polariton current state switches to the vortex state depends on the shift of the pump. For the considered parameters and the ellipticity $s=0.85$ it is $\ell \approx$ 0.65 . The vortex state with the topological charge $m=-1$ and the fractional OAM $\ell \approx-0.78$ is shown in column iv in Fig. 3 as an example. In this case, the jump of the phase in the area of the density slit supplements the change of the phase beyond the slit, so the overall phase change is $2 \pi$. In the insert to Fig. $3(\mathrm{~m})$, the coordinate origin is inside the closed curve on the phase plane $(\operatorname{Re}[\Phi], \operatorname{Im}[\Phi])$.

We performed the experimental verification of the theoretically predicted formation of polariton current states. Our experimental setup is schematically shown in Fig. 4. An exciton-polariton condensate was excited in a cylindrical pillar GaAs-based microcavity of a $25-\mu \mathrm{m}$ diameter by a nonresonant pumping at temperature of $4 \mathrm{~K}$. We use a multiple QW sample in order to maximize the exciton-photon coupling strength and achieve sizable values of the vacuumfield Rabi splitting (polariton splitting). The polariton splitting in microcavities is known to increase proportionally to the square root of the number of QWs placed in the antinodes of the optical field of the cavity mode [1]. We have measured the near-field photoluminescence spectra and compared them with interferometry images obtained with use of the MachZehnder interferometer where a spherical wave obtained by magnification of a small periphery part of the condensate image was used as a reference beam. More details on the experimental setup are given in [26]. The experimental evidence of the realization of polariton condensates with controllable fractional OAM is shown in Fig. 5. The images of the crescent polariton density distribution, Figs. 5(a) and 5(d), and the interferometry images, Figs. 5(c) and 5(f), are adapted from [43]. Two polariton condensates with opposite OAM of $\ell \approx 0.5$ [Figs. 5(a)-5(c)], and $\ell \approx-0.5$ [Figs. 5(d)-5(f)], were experimentally observed. One can conclude that circular superfluid currents exist based on the observed gradient of the phase of the condensate in the azimuthal direction. The phase (a)

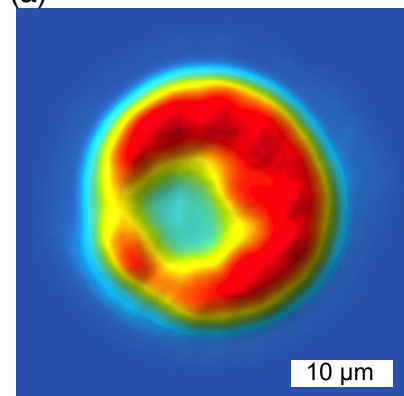

(b)

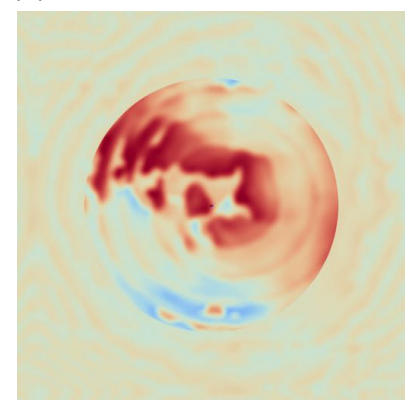

(c)
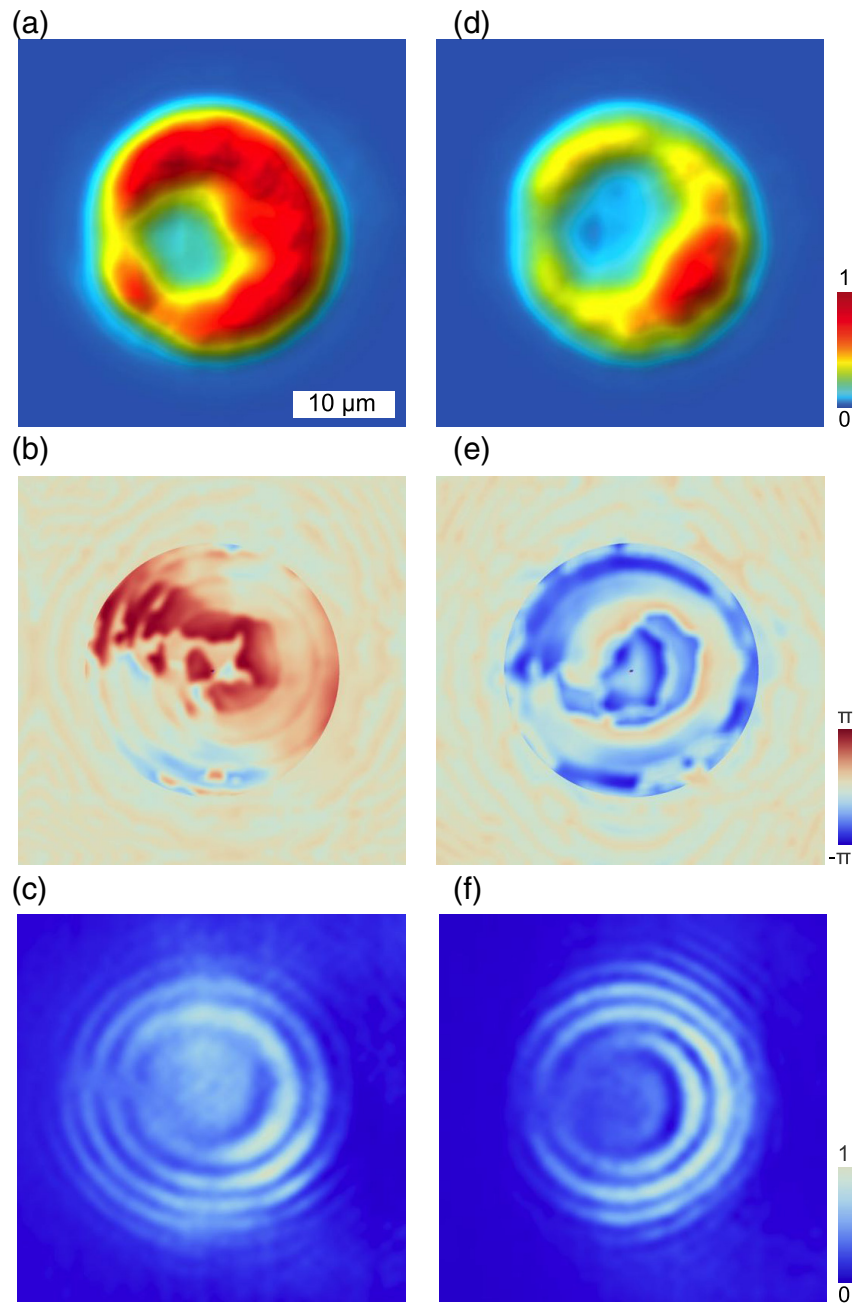

(e)

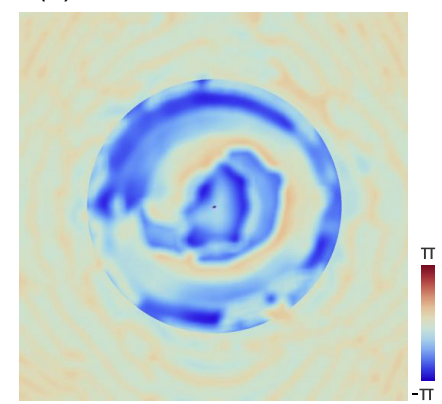

(f)

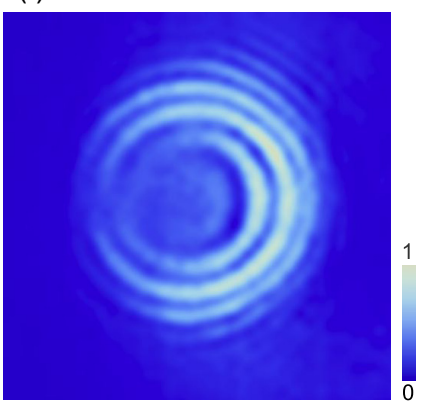

FIG. 5. The experimentally measured spatial distribution of the density (upper panels) and the phase (middle panels), and the interferometry images (lower panels) of the polariton condensates with OAM $\ell \approx 0.5$ (a)-(c) and $\ell \approx-0.5$ (d)-(f). Images (a), (c), (d), and (f) are adapted from [43]. The size bar in (a) applies to all panels.

varies with $\theta$ within a range of the width of about $\pi$ accompanied by the jump of about $\pi$ at the position of the deep in the polariton density to compensate the phase change. For the state with $\ell \approx 0.5(\ell \approx-0.5)$, phase increases (decreases) with $\theta$ beyond the density deep, while the jump is by about $-\pi(+\pi)$. The switch from one state to another was achieved by shifting the pump spot from the center of the pillar towards its edge.

\section{POLARITON VORTICES}

An ellipticity of the pump spot together with a shift of the pump spot from the center of the pillar strongly affects the azimuthal distribution of the condensate density, which significantly influences the distribution of polariton flows and causes fractional OAM. Nevertheless, even a small ellipticity $(s \rightarrow 1)$ and a small shift of tens of nanometers effectively breaks the azimuthal symmetry and induces formation of polariton vortices. Herewith the azimuthal density distribution 
(a)

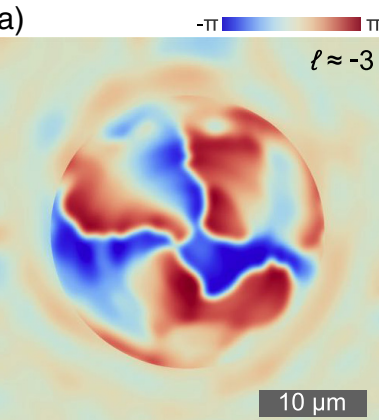

(c)

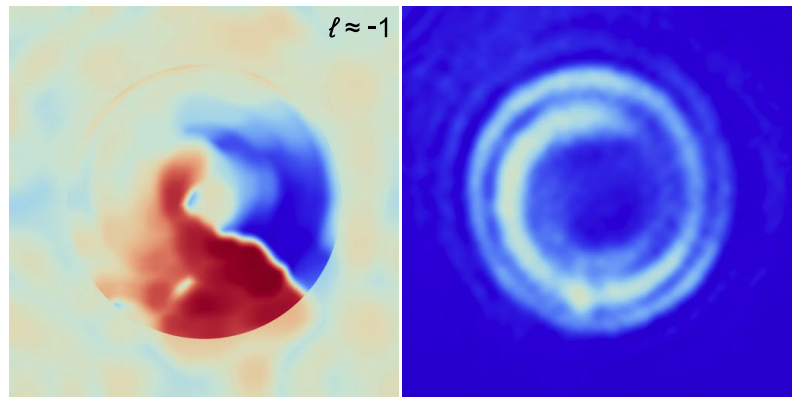

(e)
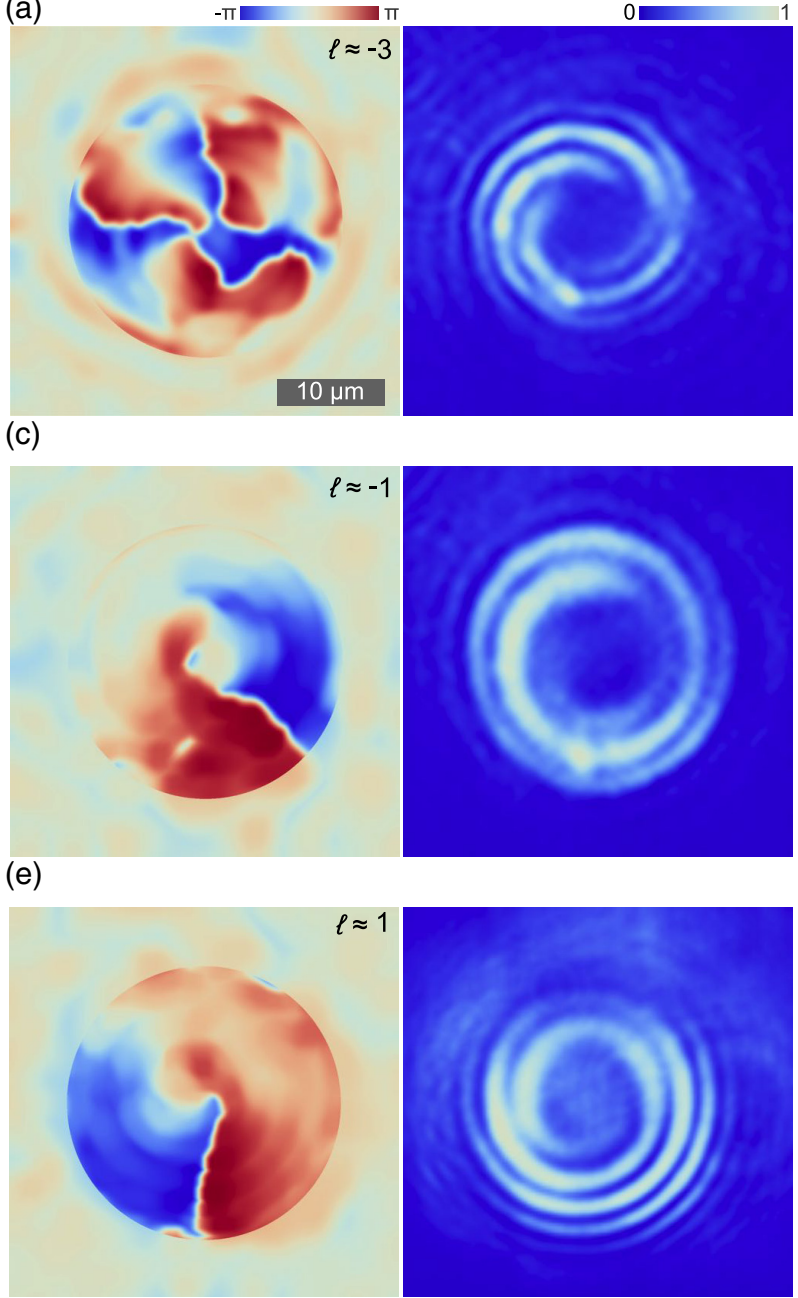

(b)
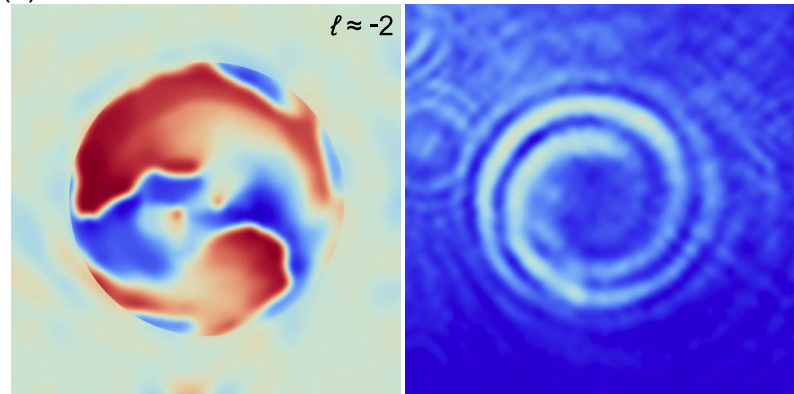

(d)

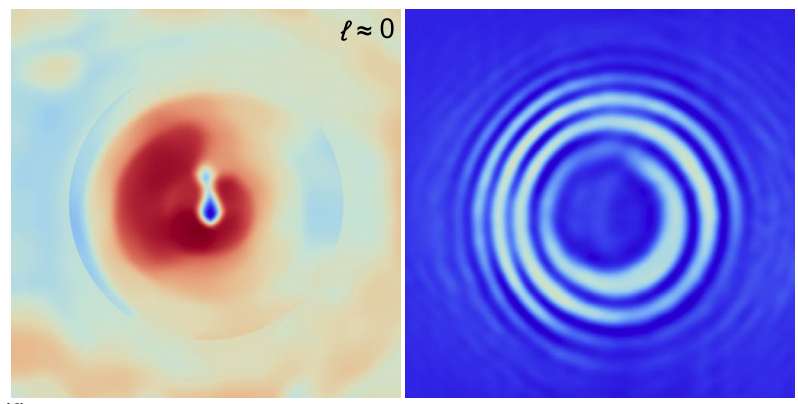

(f)

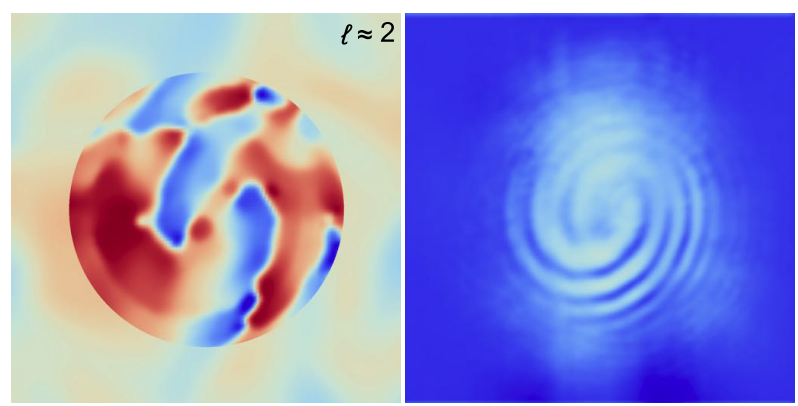

FIG. 6. The experimental spatial distributions of the phase (left panels in each pair) and the interferometry images (right panels in each pair) measured for the polariton states with integer OAM: $\ell \approx-3$ (a), $\ell \approx-2$ (b), $\ell \approx-1$ (c), $\ell \approx 0$ (d), $\ell \approx 1$ (e), $\ell \approx 2$ (f). Interferometry images on panels (d)-(f) are adapted from [26]. The size bar in (a) applies to all panels.

remains almost homogeneous along the azimuthal angle, and OAM remains nearly integer. A similar method of controllable excitation of polariton vortices has been first discussed in Ref. [26].

Figure 6 shows the spatial distributions of the phases (left images in each panel) extracted from the interferometry images (right images in each panel) obtained from the interference of the photoluminescence from the condensates with the reference spherical wave for polariton condensates with integer OAM $\ell$ varying from -3 to +2 . The interferometry images for the states with $\ell \approx 0,1,2$ are adapted from [26].

Using the proposed model, we have simulated the observed integer OAM states. The results of simulations are presented in Fig. 7. The spatial distributions of the phase density very well coincide qualitatively with the corresponding experimentally observed ones. We have supplemented the phase distributions with the polariton current density field $\mathbf{J}$. The phase varies with the azimuthal angle nearly linearly in the range of $[0,2 \pi \ell]$. The simulations confirm that for nearly integer OAM vortex states, the topological charge $m$ coincides with OAM $\ell$ in each case.

\section{DISCUSSION}

In the present work, we have demonstrated the control over the superfluid circular currents of exciton polaritons appearing in a system of a ring geometry with the controlled chirality. To excite exciton polaritons in a cylindrical pillar we used pumping with the nonresonant optical beam. This excitation method excludes the possibility of a transfer of OAM from the pump beam to the condensate or the conversion of the circular polarization of the pump to OAM of the polariton condensate via a spin-orbit interaction mechanism as in [56]. We would like to mention, that in the recent paper [57] the direct transfer of OAM from the pump beam to the polariton condensate was reported without disclosing the mechanisms of the transfer. Nevertheless, we are confident that no OAM was transferred in our case since the pump beam was Gaussian and it was not deliberately endowed with OAM. We did not detect any traces of the OAM in a pump beam emerging spontaneously. It is important to underline that the polariton condensate with no circular currents appears in the experiment much often than those with $\ell \neq 0$. The polarization measurements discussed in 
(a)

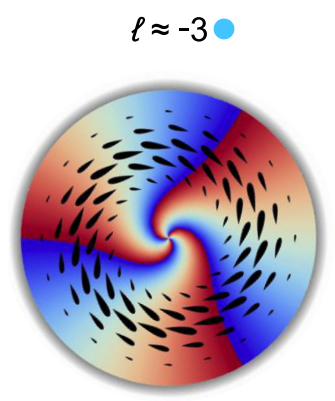

(e)

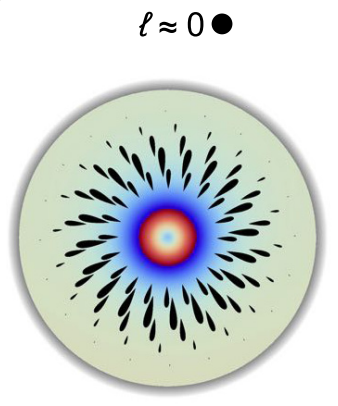

(b)

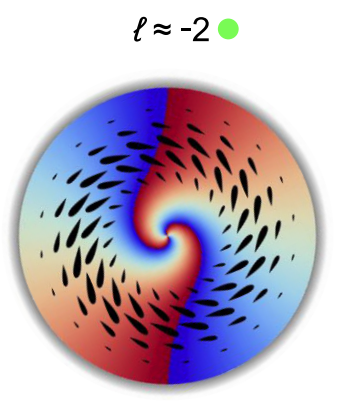

(f)

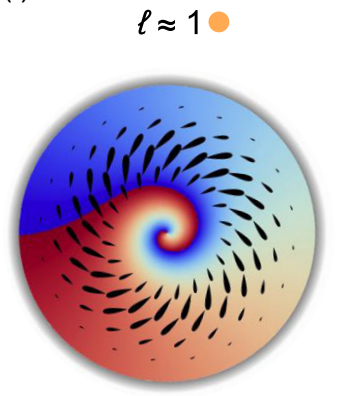

(c)

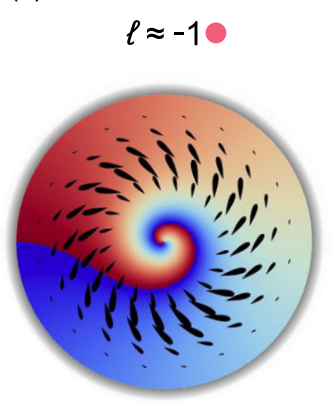

(g)

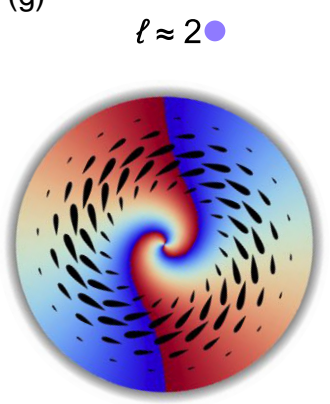

(d)

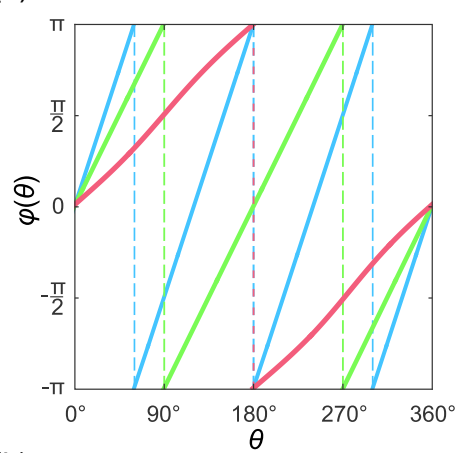

(h)

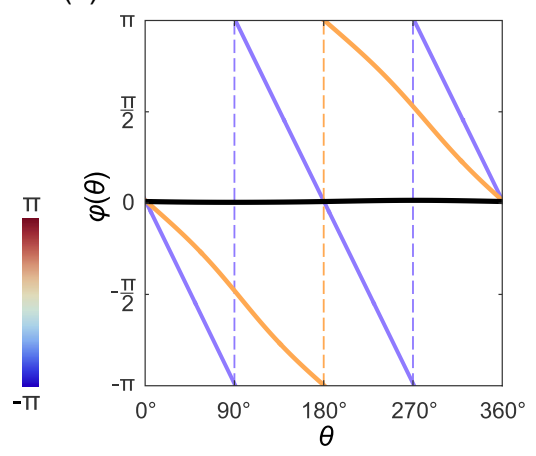

FIG. 7. The simulated spatial distributions of the phase of the condensate (a)-(c) and (e)-(g), and the variation of the phase around the pillar (d) and (h) refer to the polariton states with an integer OAM: $\ell \approx-3$ (a), $\ell \approx-2$ (b), $\ell \approx-1$ (c), $\ell \approx 0$ (e), $\ell \approx 1$ (f), $\ell \approx 2$ (g). The colors of lines in (d) and (h) coincide with colors of markers in the corresponding panels (a) $-(\mathrm{c})$ and $(\mathrm{e})-(\mathrm{g})$.

[43] also allow one to rule out the effect of the polarization of the pump beam.

We were slightly shifting the pump beam for switching between the polariton current states. Since the shift of the beam with respect to the center of the pillar in the experiment was less or comparable with the accuracy of movements of the coordinate table, we were unable to precisely control the position of the pump spot. Based on the results of simulations, we presume that the pump spot was shifted to the northeast for $\ell \approx 0.5$ and to the southeast for $\ell \approx-0.5$.

In our experiments, the system acquires chirality due to the shift of the pump. We do not certainly know what triggered this in the experiment in addition to the pump spot shift. Among the possible reasons are the defect of the stationary potential from the pillar edge, the broken azimuthal symmetry of the pump beam or the pillar. All these options can be easily taken into account in the proposed model.

The established states both with integer and fractional OAM are highly stable and reproducible. Once emerged, they exist until the pump is on. In this regime, the OAM of the condensate is being preserved. After switching the pump off and on, OAM of the established ring current turns to be unchanged. The stability of the states and the conservation of both OAM $\ell$ and the topological charge $m$ bring us to the conclusion that the observed states are eigenstates of the nonlinear system. In this connection, the expansion (9) should be understood not as the linear superposition of quantum topological states, but referred to as the set of nonlinear states with superimposed complex spectral coefficients $\psi_{m}$. The spatial coherence of the resulting state is kept constant during the time of the experimental measurement, while the overall timedependent phase of the multicomponent wave function of the condensate may be subject to dephasing. The expansion (9) is analogous to the Fourier transform of a function with periodic boundary conditions in this case.

We have demonstrated that superfluid circular currents of polaritons do not necessarily indicate formation of vortices in a polariton condensate. The distinction between the current and vortex states is especially evident in the approach we use for controlling a polariton condensate behavior, that is, the reservoir engineering. Polariton currents originate from imbalance in real space of polariton inflow and outflow induced by a spatially localized pump. Trapped in a confinement potential they acquire a nonzero OAM determined by the azimuthal variation of both the density and the phase of the polariton condensate. A vortex state of a polariton condensate is a particular current state characterized by the nonzero azimuthal variation of its phase which changes by an integer number of $2 \pi$ when going around the ring. The variation of the phase of the condensate is reflected in the helical wave front of the photoluminescence emission from the condensate. The topological charge is the measure of the helicity of emitted light. By optically controlling the effective complex potential, we have demonstrated in our experiment excitation of circular current states without vorticity.

In the present manuscript, we have developed the numerical model for simulating the experimentally observed polariton states both with fractional and integer OAM. Factorizing the wave function of the condensate, we limited ourselves to considering the ground state of the radial 
component. Let us now list the main arguments in favor of this assumption. In the ring geometry, the narrower the ring, the better justified is separation of the azimuthal and radial components of the wave function. In the limit of an infinitely narrow ring, only the ground radial state exists that is characterized by an infinite set of azimuthal states. In a ring of a finite width, the sequence of states with $n \geqslant 1$ and $m \neq 0$ on the energy scale is governed by the shape of the potential. In the expansion on a narrow ring, several lower energy states can be characterized by $n=1$ and differ only by the value of $m$. The narrower the ring, the more states with $n=1$ and different $m$ join the bunch of lower energy states. Our simulations show that for the used parameters the maximal azimuthal quantum number $|m|$ for the ground radial state exceeds with a margin the largest substantially contributing $m$ state in the expansion of the fractional OAM states. Another argument for the validity of this assumption is that in the given geometry of the pillar and the pump beam no other radial states than the ground state have been observed in our experiments.

Finally, we would like to comment on the observability and measurement of fractional OAM states. An elegant method of measuring fractional OAM of light was proposed in the recent paper [58]. The method is based on decomposing the light beam into a series of integer vortex beams in real space. The OAM of the beam is determined by averaging the multiplexing beams with their energies as the weighting coefficients.
In this sense, the decomposition (9) represents a convenient mathematical model that is embodied in the experimental instrument.

\section{ACKNOWLEDGMENTS}

This work is from the Innovative Team of International Center for Polaritonics and is supported by Westlake University (Project No. 041020100118). E.S.S. and A.V.K. acknowledge support from Program No. 2018R01002 funded by Leading Innovative and Entrepreneur Team Introduction Program of Zhejiang. The study was done with the support of the state assignment of VlSU within the framework of the Project No. 0635-2020-0013, the Russian Foundation for Basic Research (RFBR Project No. 21-52-10005), and the Grant of the President of the Russian Federation for state support of young Russian scientists (Grant No. MK-4729.2021.1.2). V.A.L., V.K.K., and A.V.K. acknowledge financial support from RFBR Project No. 19-52-12032 and Saint Petersburg State University for Research Grant No. 28874264. A.V.K. acknowledges support from Rosatom within the Road Map for Quantum Computing. The authors acknowledge Professor N. Gippius, Professor S. Tikhodeev, and Dr. I. Chestnov for fruitful discussions, critical evaluation of the work, and sharing their ideas on the development of the study.
[1] A. Kavokin, J. Baumberg, G. Malpuech, and F. Laussy, Microcavities, 2nd ed., Series on Semiconductor Science and Technology (Oxford University Press, Oxford, 20017).

[2] D. Sanvitto, F. M. Marchetti, M. H. Szymańska, G. Tosi, M. Baudisch, F. P. Laussy, D. N. Krizhanovskii, M. S. Skolnick, L. Marrucci, A. Lemaître, J. Bloch, C. Tejedor, and L. Viña, Persistent currents and quantized vortices in a polariton superfluid, Nat. Phys. 6, 527 (2010).

[3] I. Carusotto and C. Ciuti, Quantum fluids of light, Rev. Mod. Phys. 85, 299 (2013).

[4] L. Deák and T. Fülöp, Reciprocity in quantum, electromagnetic and other wave scattering, Ann. Phys. 327, 1050 (2012).

[5] A. Ramanathan, K. C. Wright, S. R. Muniz, M. Zelan, W. T. Hill, C. J. Lobb, K. Helmerson, W. D. Phillips, and G. K. Campbell, Superflow in a Toroidal Bose-Einstein Condensate: An Atom Circuit with a Tunable Weak Link, Phys. Rev. Lett. 106, 130401 (2011).

[6] M. Cominotti, D. Rossini, M. Rizzi, F. Hekking, and A. Minguzzi, Optimal Persistent Currents for Interacting Bosons on a Ring with a Gauge Field, Phys. Rev. Lett. 113, 025301 (2014).

[7] D. Aghamalyan, M. Cominotti, M. Rizzi, D. Rossini, F. Hekking, A. Minguzzi, L.-C. Kwek, and L. Amico, Coherent superposition of current flows in an atomtronic quantum interference device, New J. Phys. 17, 045023 (2015).

[8] J. Dalibard, F. Gerbier, G. Juzeliūnas, and P. Öhberg, Colloquium: Artificial gauge potentials for neutral atoms, Rev. Mod. Phys. 83, 1523 (2011).

[9] N. Goldman, G. Juzeliūnas, P. Öhberg, and I. B. Spielman, Light-induced gauge fields for ultracold atoms, Rep. Prog. Phys. 77, 126401 (2014).
[10] A. L. Fetter, Rotating trapped bose-einstein condensates, Rev. Mod. Phys. 81, 647 (2009).

[11] M. Hafezi and P. Rabl, Optomechanically induced nonreciprocity in microring resonators, Opt. Express 20, 7672 (2012).

[12] Z. Yu and S. Fan, Complete optical isolation created by indirect interband photonic transitions, Nat. Photonics 3, 91 (2009).

[13] M. Soljačić, C. Luo, J. D. Joannopoulos, and S. Fan, Nonlinear photonic crystal microdevices for optical integration, Opt. Lett. 28, 637 (2003).

[14] G. Li, M. D. Fraser, A. Yakimenko, and E. A. Ostrovskaya, Stability of persistent currents in open dissipative quantum fluids, Phys. Rev. B 91, 184518 (2015).

[15] T. Gao, E. Estrecho, K. Y. Bliokh, T. C. H. Liew, M. D. Fraser, S. Brodbeck, M. Kamp, C. Schneider, S. Höfling, Y. Yamamoto, F. Nori, Y. S. Kivshar, A. G. Truscott, R. G. Dall, and E. A. Ostrovskaya, Observation of non-Hermitian degeneracies in a chaotic exciton-polariton billiard, Nature (London) 526, 554 (2015).

[16] A. Metelmann and A. A. Clerk, Nonreciprocal Photon Transmission and Amplification Via Reservoir Engineering, Phys. Rev. X 5, 021025 (2015).

[17] M. Keck, D. Rossini, and R. Fazio, Persistent currents by reservoir engineering, Phys. Rev. A 98, 053812 (2018).

[18] C. Wang and J. M. Gertler, Autonomous quantum state transfer by dissipation engineering, Phys. Rev. Research 1, 033198 (2019).

[19] M. Aßmann, F. Veit, M. Bayer, A. Löffler, S. Höfling, M. Kamp, and A. Forchel, All-optical control of quantized momenta on a polariton staircase, Phys. Rev. B 85, 155320 (2012). 
[20] K. P. Kalinin and N. G. Berloff, Toward arbitrary control of lattice interactions in nonequilibrium condensates, Adv. Quantum Technol. 3, 1900065 (2020).

[21] N. Stroev and N. G. Berloff, Managing flow of liquid light, Phys. Rev. B 102, 201114(R) (2020).

[22] A. S. Desyatnikov, Y. S. Kivshar, and L. Torner, in Optical Vortices and Vortex Solitons (Elsevier, Amsterdam, 2005), pp. 291-391.

[23] A. V. Carpentier, H. Michinel, J. R. Salgueiro, and D. Olivieri, Making optical vortices with computer-generated holograms, Am. J. Phys. 76, 916 (2008).

[24] R. Dall, M. D. Fraser, A. S. Desyatnikov, G. Li, S. Brodbeck, M. Kamp, C. Schneider, S. Höfling, and E. A. Ostrovskaya, Creation of Orbital Angular Momentum States with Chiral Polaritonic Lenses, Phys. Rev. Lett. 113, 200404 (2014).

[25] N. Carlon Zambon, P. St-Jean, M. Milićević, A. Lemaître, A. Harouri, L. Le Gratiet, O. Bleu, D. D. Solnyshkov, G. Malpuech, I. Sagnes, S. Ravets, A. Amo, and J. Bloch, Optically controlling the emission chirality of microlasers, Nat. Photonics 13, 283 (2019).

[26] V. A. Lukoshkin, V. K. Kalevich, M. M. Afanasiev, K. V. Kavokin, Z. Hatzopoulos, P. G. Savvidis, E. S. Sedov, and A. V. Kavokin, Persistent circular currents of exciton-polaritons in cylindrical pillar microcavities, Phys. Rev. B 97, 195149 (2018).

[27] S. Eckel, F. Jendrzejewski, A. Kumar, C. J. Lobb, and G. K. Campbell, Interferometric Measurement of the Current-Phase Relationship of a Superfluid Weak Link, Phys. Rev. X 4, 031052 (2014).

[28] O. L. Berman, R. Y. Kezerashvili, G. V. Kolmakov, and L. M. Pomirchi, Spontaneous formation and nonequilibrium dynamics of a soliton-shaped Bose-Einstein condensate in a trap, Phys. Rev. E 91, 062901 (2015).

[29] M. V. Berry, Optical vortices evolving from helicoidal integer and fractional phase steps, Journal of Optics A: Pure and Applied Optics 6, 259 (2004).

[30] J. Leach, E. Yao, and M. J. Padgett, Observation of the vortex structure of a non-integer vortex beam, New J. Phys. 6, 71 (2004).

[31] S. Vyas, R. K. Singh, and P. Senthilkumaran, Fractional vortex lens, Opt. Laser Technol. 42, 878 (2010).

[32] S. S. R. Oemrawsingh, J. A. W. van Houwelingen, E. R. Eliel, J. P. Woerdman, E. J. K. Verstegen, J. G. Kloosterboer, and G. W. 't Hooft, Production and characterization of spiral phase plates for optical wavelengths, Appl. Opt. 43, 688 (2004).

[33] Y. G. Rubo, Half Vortices in Exciton Polariton Condensates, Phys. Rev. Lett. 99, 106401 (2007).

[34] K. G. Lagoudakis, T. Ostatnický, A. V. Kavokin, Y. G. Rubo, R. André, and B. Deveaud-Plédran, Observation of half-quantum vortices in an exciton-polariton condensate, Science 326, 974 (2009).

[35] F. Manni, K. G. Lagoudakis, T. C. H. Liew, R. André, V. Savona, and B. Deveaud, Dissociation dynamics of singly charged vortices into half-quantum vortex pairs, Nat. Commun. 3, 1309 (2012).

[36] L. Dominici, G. Dagvadorj, J. M. Fellows, D. Ballarini, M. De Giorgi, F. M. Marchetti, B. Piccirillo, L. Marrucci, A. Bramati, G. Gigli, M. H. Szymańska, and D. Sanvitto, Vortex and halfvortex dynamics in a nonlinear spinor quantum fluid, Sci. Adv. 1, e1500807 (2015).
[37] G. Liu, D. W. Snoke, A. Daley, L. N. Pfeiffer, and K. West, A new type of half-quantum circulation in a macroscopic polariton spinor ring condensate, Proc. Natl. Acad. Sci. 112, 2676 (2015).

[38] D. Schmidt, B. Berger, M. Kahlert, M. Bayer, C. Schneider, S. Höfling, E. S. Sedov, A. V. Kavokin, and M. Aßmann, Tracking Dark Excitons with Exciton Polaritons in Semiconductor Microcavities, Phys. Rev. Lett. 122, 047403 (2019).

[39] J. Keeling and N. G. Berloff, Spontaneous Rotating Vortex Lattices in a Pumped Decaying Condensate, Phys. Rev. Lett. 100, 250401 (2008).

[40] F. E. Meijer, A. F. Morpurgo, and T. M. Klapwijk, One-dimensional ring in the presence of Rashba spin-orbit interaction: Derivation of the correct Hamiltonian, Phys. Rev. B 66, 033107 (2002)

[41] S. Bargi, F. Malet, G. M. Kavoulakis, and S. M. Reimann, Persistent currents in bose gases confined in annular traps, Phys. Rev. A 82, 043631 (2010).

[42] We take the following parameters for simulations. The effective mass of polaritons is $M=5 \times 10^{-5} m_{\mathrm{e}}$, where $m_{\mathrm{e}}$ is the free electron mass. The polariton and exciton decay rates are taken as $\gamma_{\mathrm{C}}=0.02 \mathrm{ps}^{-1}$ and $\gamma_{\mathrm{X}}=0.025 \mathrm{ps}^{-1}$, respectively. The stimulated scattering rate is taken as $\hbar R=0.05 \mathrm{meV}_{\mu} \mathrm{m}^{2}$. The nonlinearity coefficients are taken as $\alpha=\alpha_{\mathrm{R}} / 2=3 \mu \mathrm{eV} \mu \mathrm{m}^{2}$. The pump width is $w_{\mathrm{p}}=2 \mu \mathrm{m}$. The diameter of the pillar is $25 \mu \mathrm{m}$.

[43] E. Sedov, V. Lukoshkin, V. Kalevich, Z. Hatzopoulos, P. Savvidis, and A. Kavokin, Persistent currents in half-moon polariton condensates, ACS Photonics 7, 1163 (2020).

[44] E. A. Ostrovskaya, J. Abdullaev, A. S. Desyatnikov, M. D. Fraser, and Y. S. Kivshar, Dissipative solitons and vortices in polariton Bose-Einstein condensates, Phys. Rev. A 86, 013636 (2012).

[45] V. K. Kalevich, M. M. Afanasiev, V. A. Lukoshkin, D. D. Solnyshkov, G. Malpuech, K. V. Kavokin, S. I. Tsintzos, Z. Hatzopoulos, P. G. Savvidis, and A. V. Kavokin, Controllable structuring of exciton-polariton condensates in cylindrical pillar microcavities, Phys. Rev. B 91, 045305 (2015).

[46] Y. Xue, I. Chestnov, E. Sedov, S. Schumacher, X. Ma, and A. Kavokin, Split-ring polariton condensates as macroscopic twolevel quantum systems, arXiv:1907.00383v4.

[47] B. Nelsen, G. Liu, M. Steger, D. W. Snoke, R. Balili, K. West, and L. Pfeiffer, Dissipationless Flow and Sharp Threshold of a Polariton Condensate with Long Lifetime, Phys. Rev. X 3 , 041015 (2013).

[48] D. M. Myers, B. Ozden, M. Steger, E. Sedov, A. Kavokin, K. West, L. N. Pfeiffer, and D. W. Snoke, Superlinear increase of photocurrent due to stimulated scattering into a polariton condensate, Phys. Rev. B 98, 045301 (2018).

[49] T. Boulier, H. Terças, D. D. Solnyshkov, Q. Glorieux, E. Giacobino, G. Malpuech, and A. Bramati, Vortex chain in a resonantly pumped polariton superfluid, Sci. Rep. 5, 9230 (2015).

[50] D. M. Myers, J. K. Wuenschell, B. Ozden, J. Beaumariage, D. W. Snoke, L. Pfeiffer, and K. West, Edge trapping of excitonpolariton condensates in etched pillars, Appl. Phys. Lett. 110, 211104 (2017).

[51] E. Wertz, L. Ferrier, D. D. Solnyshkov, R. Johne, D. Sanvitto, A. Lemaître, I. Sagnes, R. Grousson, A. V. Kavokin, P. Senellart, G. Malpuech, and J. Bloch, Spontaneous formation and optical manipulation of extended polariton condensates, Nat. Phys. 6 , 860 (2010) 
[52] M. Sich, L. E. Tapia-Rodriguez, H. Sigurdsson, P. M. Walker, E. Clarke, I. A. Shelykh, B. Royall, E. S. Sedov, A. V. Kavokin, D. V. Skryabin, M. S. Skolnick, and D. N. Krizhanovskii, Spin domains in one-dimensional conservative polariton solitons, ACS Photonics 5, 5095 (2018).

[53] J. Schmutzler, P. Lewandowski, M. Aßmann, D. Niemietz, S. Schumacher, M. Kamp, C. Schneider, S. Höfling, and M. Bayer, All-optical flow control of a polariton condensate using nonresonant excitation, Phys. Rev. B 91, 195308 (2015).

[54] A. Askitopoulos, A. V. Nalitov, E. S. Sedov, L. Pickup, E. D. Cherotchenko, Z. Hatzopoulos, P. G. Savvidis, A. V. Kavokin, and P. G. Lagoudakis, All-optical quantum fluid spin beam splitter, Phys. Rev. B 97, 235303 (2018).

[55] B. Berger, D. Schmidt, X. Ma, S. Schumacher, C. Schneider, S. Höfling, and M. Aßmann, Formation dynamics of exciton- polariton vortices created by nonresonant annular pumping, Phys. Rev. B 101, 245309 (2020).

[56] F. Manni, K. G. Lagoudakis, T. K. Paraïso, R. Cerna, Y. Léger, T. C. H. Liew, I. A. Shelykh, A. V. Kavokin, F. Morier-Genoud, and B. Deveaud-Plédran, Spin-to-orbital angular momentum conversion in semiconductor microcavities, Phys. Rev. B 83, 241307(R) (2011).

[57] M.-S. Kwon, B. Y. Oh, S.-H. Gong, J.-H. Kim, H. K. Kang, S. Kang, J. D. Song, H. Choi, and Y.-H. Cho, Direct Transfer of Light's Orbital Angular Momentum onto a Nonresonantly Excited Polariton Superfluid, Phys. Rev. Lett. 122, 045302 (2019).

[58] D. Deng, M. Lin, Y. Li, and H. Zhao, Precision Measurement of Fractional Orbital Angular Momentum, Phys. Rev. Applied 12, 014048 (2019). 